\title{
Variz pulmonar: A propósito de un caso y revisión de la literatura
}

\author{
Marco Antonio Díaz E. ${ }^{*}$, Novar López C. ${ }^{2}$, Fabiola García S. ${ }^{3}$, Abril Maciel F.4, Jair Díaz E. ${ }^{5}$
}

1. Residente de cuarto año de imagenología del Hospital de Especialidades del Centro Médico Nacional Siglo XXI. Ciudad de México, México.

2. Residente de tercer año de imagenología del Hospital de Especialidades del Centro Médico Nacional Siglo XXI. Ciudad de México, México.

3. Residente de segundo año de imagenología del Hospital de Especialidades del Centro Médico Nacional Siglo XXI. Ciudad de México, México.

4. Médico adscrito al servicio de imagenología del Hospital de Especialidades del Centro Médico Nacional Siglo XXI. Ciudad de México, México.

5. Médico de tercer año de pregrado de la escuela superior de medicina del Instituto Politécnico Nacional. Ciudad de México, México.

\section{Pulmonary Varix: A case report and review of the literature}

\section{Resumen:}

La variz venosa pulmonar es una entidad rara, definida como una dilatación patológica focal de una vena pulmonar que puede ser congénita o adquirida. Dichas dilataciones venosas no tienen predominio de género y pueden ocurrir a cualquier edad, su incidencia es desconocida pero se han informado con poca frecuencia. Clínicamente suelen ser asintomáticas, siendo hallazgos incidentales en la radiografía en donde pueden imitar una masa mediastínica parahiliar o parenquimatosa. La tomografía contrastada de tórax es de mayor utilidad para el diagnóstico al igual que la angiografía y la resonancia magnética. El tratamiento es innecesario en la mayoría de los casos y el pronóstico suele ser bueno. Presentamos el caso de un paciente con dilatación varicosa de la vena pulmonar diagnosticada incidentalmente con una revisión de la literatura sobre el tema.

Palabras clave: Diagnóstico imagenológico; Variz venosa; Venas pulmonares.

\begin{abstract}
:
Pulmonary varix is a rare entity, defined as a focal pathological dilation of a pulmonary vein that can be congenital or acquired. These venous dilations do not have a gender predominance and can occur at any age, their incidence is unknown but they have been reported infrequently. Clinically they are usually asymptomatic, being discovered incidentally on radiography where they can mimic a parahilar or parenchymal mediastinal mass. Contrast-enhanced chest tomography is more useful for diagnosis than angiography and magnetic resonance imaging. Treatment is unnecessary in most cases and the prognosis is usually good. We present the case of a patient with varicose dilation of the pulmonary vein diagnosed incidentally with a review of the literature on the subject.
\end{abstract}

Keywords: Diagnostic imaging; Pulmonary veins; Varicose veins.

Díaz MA, et al. Variz pulmonar: A propósito de un caso y revisión de la literatura. Rev Chil Radiol 2021; 27(2): $104-108$. *Correspondencia: Marco Antonio Díaz Escareño / mdiazesc@gmail.com

Trabajo enviado el 05 de agosto de 2020. Aceptado para publicación el 04 de octubre de 2020. 


\section{Introducción}

La variz venosa pulmonar es una entidad rara, descrita originalmente en 1843 por Puchet en la autopsia de un niño. Se define como una dilatación patológica focal de una vena pulmonar que puede ser congénita o adquirida. No tienen predominio de género y pueden ocurrir a cualquier edad ${ }^{1,2}$. Suele representar un reto en el diagnóstico imagenológico inicial, ya que algunas veces puede llegar a confundirse con un nódulo pulmonar, lo que obliga a la realización de estudios de extensión para un diagnóstico certero.

Presentamos el caso de un paciente estudiado por patología gastrointestinal, en el cual se descubrió mediante estudios de extensión la dilatación varicosa de la vena pulmonar. Se incluye una revisión de la literatura sobre el tema.

\section{Descripción del caso}

Masculino de 60 años con antecedente de cardiopatía isquémica crónica e insuficiencia mitral leve en seguimiento actual por cardiología. Es valorado en el servicio de urgencias por presentar dolor abdominal en mesogastrio de intensidad leve, tinte ictérico, coluria y acolia. En el interrogatorio refiere además pérdida ponderal aproximada de $7 \mathrm{~kg}$ en 3 meses.

A la exploración física, el paciente se encuentra neurológicamente íntegro, hemodinámicamente estable, ruidos respiratorios presentes, primer ruido cardíaco único y segundo ruido desdoblado, abdomen sin datos de irritación peritoneal.

El estudio de resonancia magnética en conjunto con la tomografía computarizada abdominal evidenciaron una tumoración a nivel de la cabeza del páncreas con dilatación de la vía biliar intra y extrahepática, así como del conducto pancreático (Figura 1).

Se realiza estudio de CPRE (colangiopancreatografía endoscópica retrógrada), que en conjunto con el ultrasonido endoscópico confirman hallazgos de los estudios de imagen a la vez que se realiza toma de biopsia para confirmación de estirpe histológica del tumor y colocación de endoprótesis biliar.

La radiografía de tórax mostró un discreto aumento de la opacidad retro cardiaca y adyacente al perfil cardíaco derecho (Figura 2). Para una mejor evaluación se realizó tomografía de tórax contrastada, la cual mostró una dilatación sacular de la vena pulmonar inferior derecha hacia la zona de confluencia en el ventrículo izquierdo, de igual forma con dilatación de las cavidades cardiacas izquierdas (Figura 3). La vena pulmonar derecha superior de igual forma se observó levemente dilatada. En la imagen de resonancia magnética complementaria se confirma la dilatación sacular de la vena pulmonar inferior derecha (Figura 4).

El ecocardiograma demostró una insuficiencia

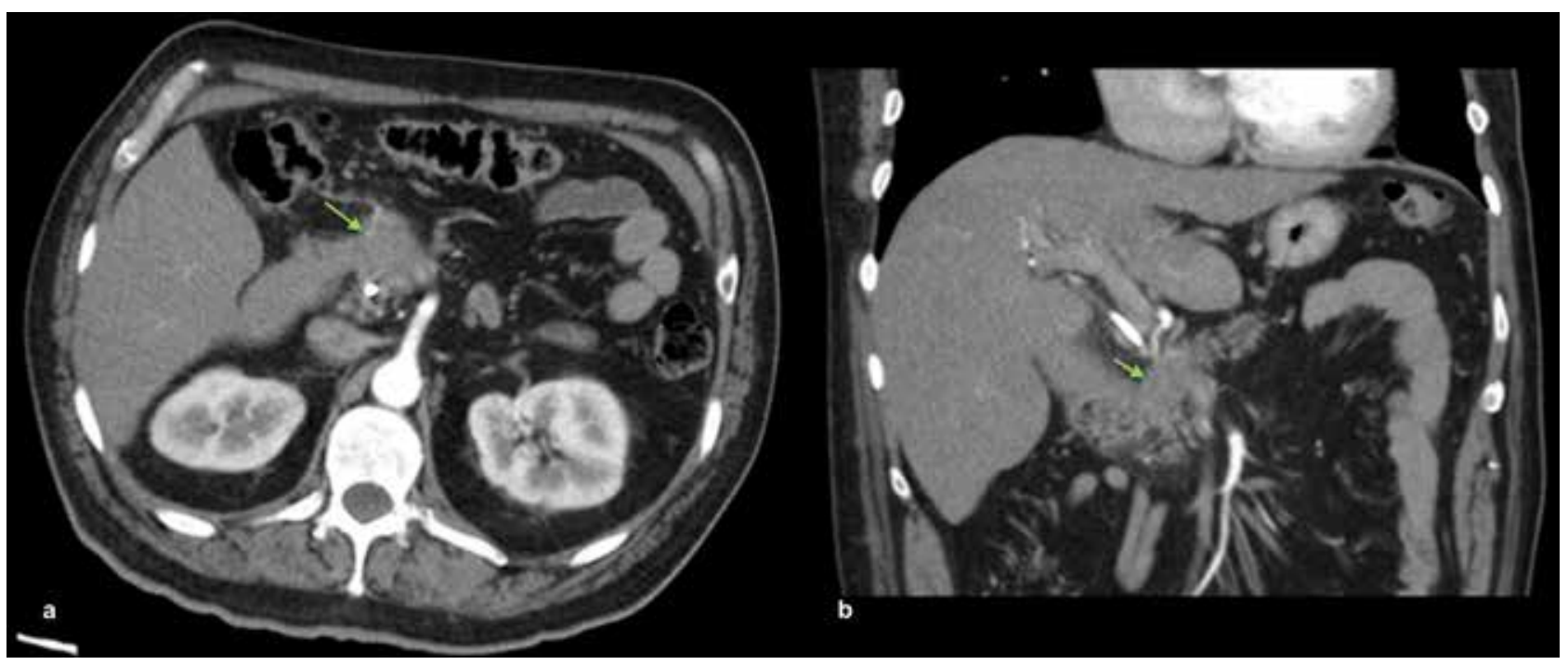

Figura 1: Imágenes de un estudio de tomografía abdominal en fase contrastada en corte axial (a) y reconstrucción en plano coronal (b). En cabeza del páncreas, se identifica lesión ovoidea, de márgenes irregulares parcialmente definidos, con el medio de contraste tiene un comportamiento levemente hipodenso en comparación con el parénquima pancreático. Compatible con lesión tumoral sugestiva de adenocarcinoma. 
mitral leve con fracción de eyección conservada, sin alteraciones de la movilidad.

El diagnóstico final fue adenocarcinoma de páncreas que requirió tratamiento quirúrgico y como hallazgo adicional, se documentó la presencia de dilatación varicosa de la vena pulmonar inferior derecha con insuficiencia mitral leve y cardiopatía isquémica crónica.

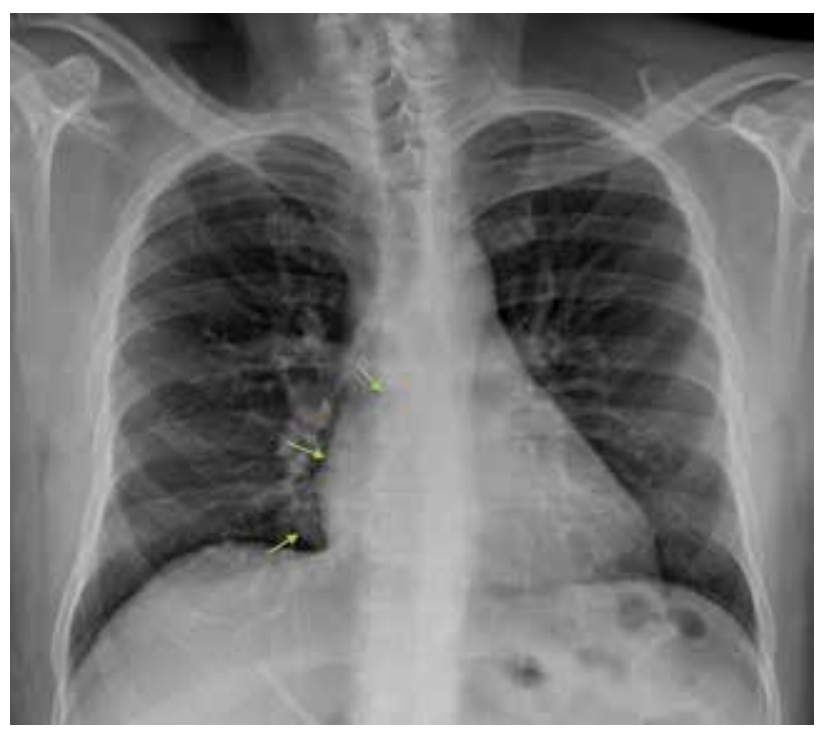

Figura 2: Radiografía de tórax en proyección $P A$. Se identifica discreto aumento de la opacidad retro- y paracardíaca derecha, de morfología ovoidea, que supone un aumento en el tamaño de las cavidades derechas mayormente a nivel auricular; sin alguna otra anormalidad pleuropulmonar significativa. Adicionalmente se identifica prótesis de situación biliar.

\section{Discusión}

Las várices pulmonares son infrecuentes y generalmente son asintomáticas, siendo hallazgos incidentales en una radiografía de tórax o una tomografía computarizada, muy raramente los pacientes presentan problemas respiratorios ${ }^{3}$. Se ha informado la presencia de hemoptsisis agudas o crónicas, pudiendo ser fatal si ocurre la ruptura de las várices en un bronquio o en el espacio pleural ocasionando hemotórax ${ }^{4}$.

Otras manifestaciones clínicas raras incluyen el síndrome del lóbulo medio derecho por compresión extrínseca, disfagia o embolia sistémica ${ }^{5}$.

Su etiología sigue siendo incierta, destacando su origen congénito 0 adquirido ${ }^{6}$. Si es congénita, puede deberse a diversas entidades como: conducto arterioso persistente permeable, defecto del tabique ventral, a una telangiectasia hemorrágica hereditaria o puede surgir en el contexto de un desarrollo venoso pulmonar anómalo. Las várices adquiridas son de origen incierto, sin embargo, tienen una fuerte asociación con anomalías valvulares cardíacas, como la enfermedad de la válvula mitral, que está presente en un tercio de los pacientes con varices adquiridas, estando de igual forma asociadas a hipertensión venosa pulmonar y hasta cirrosis hepática con hipertensión portal ${ }^{7}$.

En cuanto al diagnóstico como ya hemos mencionado suelen ser un hallazgo incidental. En la radiografía pueden imitar una masa mediastínica, perihiliar o parenquimatosa ${ }^{8}$. Se ha propuesto una clasificación basada en las características radiográficas del tórax, como son: 1. Tipo sacular. Dilatación localizada, ovalada o sacular de la vena pulmonar; 2. Tipo tortuoso: Dilatación torcida y alargada de la vena pulmonar; 3 . Tipo confluente: dilatación de la

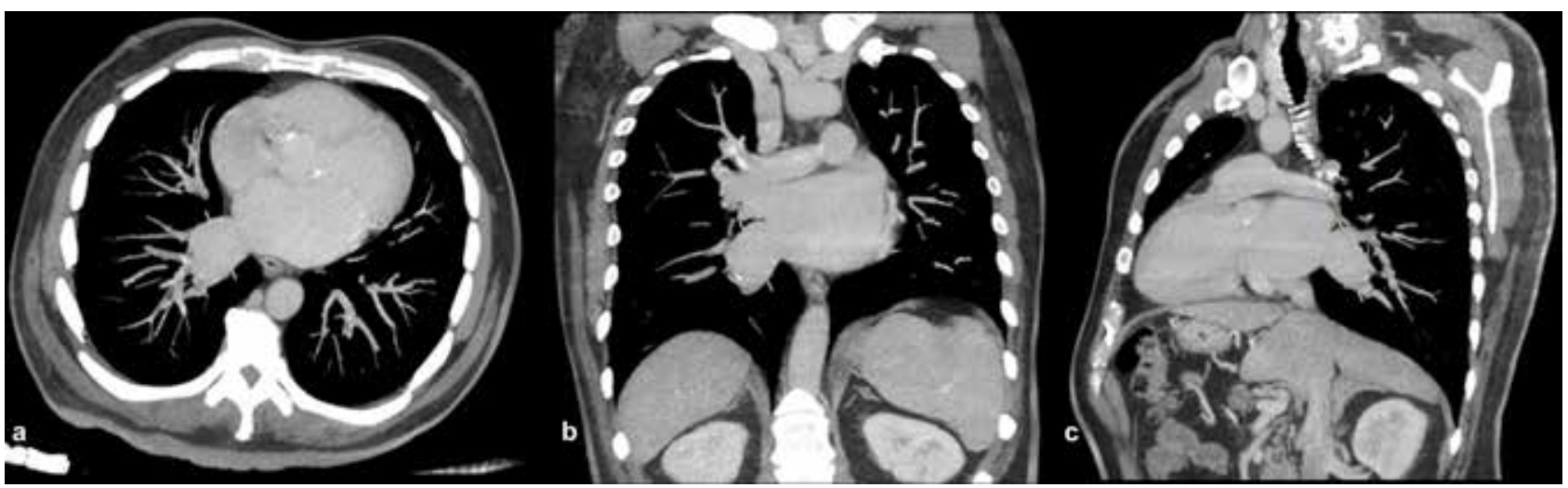

Figura 3: Imágenes en máxima intensidad de proyección (MIP) de un estudio de tomografía contrastada de tórax en corte axial (a), reconstrucción en plano coronal oblicua (b) y sagital oblicua (c). Se identifica una dilatación sacular de la vena pulmonar inferior derecha hacia la confluencia de la aurícula izquierda, de aproximadamente $30 \times 29 \times 33 \mathrm{~mm}$ en sus ejes mayores. Compatible con variz de la vena pulmonar. 


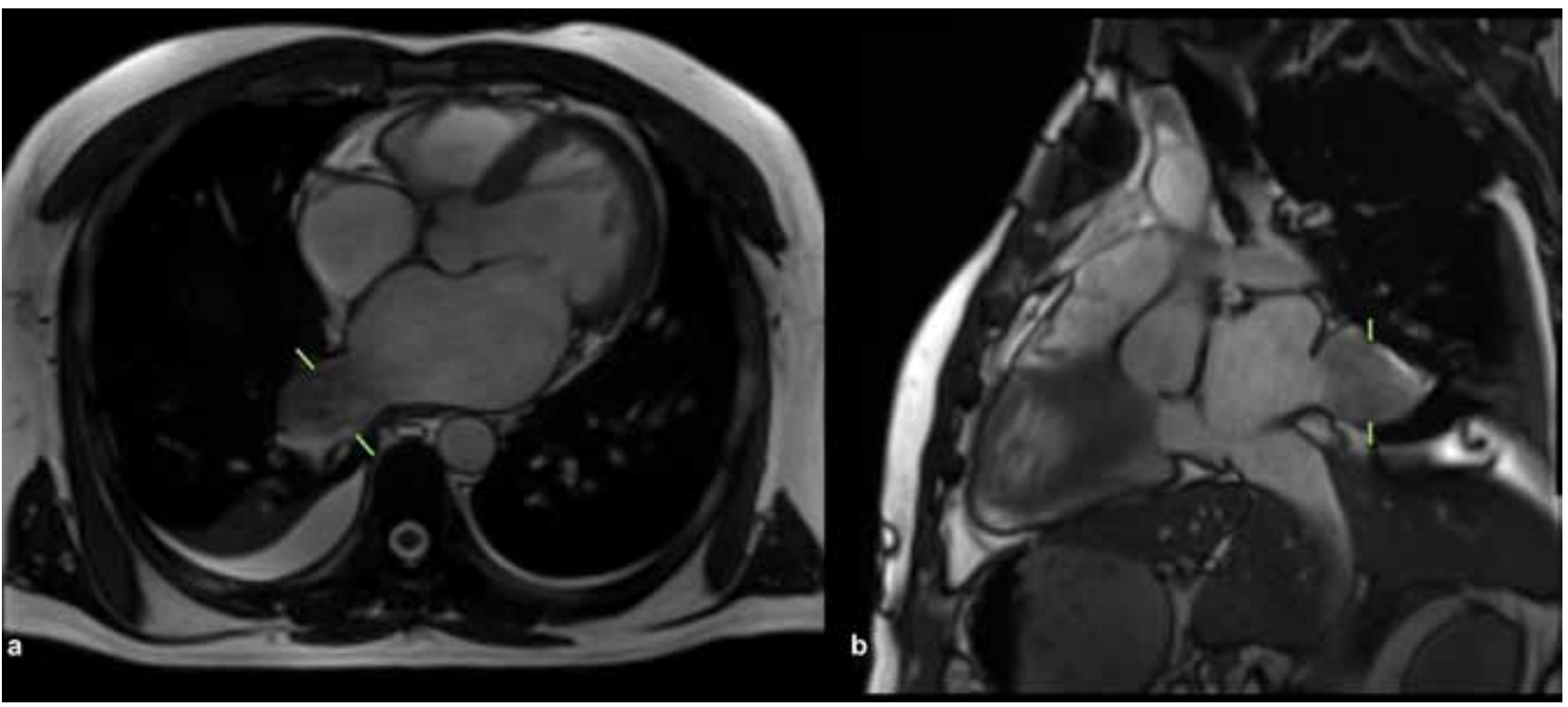

Figura 4: Imágenes de un estudio de resonancia magnética en ponderación Eco de gradiente (SSFP), corte transversal y reconstrucción sagital oblicua. Se confirma el hallazgo descrito como dilatación de la vena pulmonar inferior derecha compatible con variz pulmonar. Se observa más hipointensa que el resto de la aurícula debido al flujo turbulento generado a este nivel, dado que ésta secuencia posee alta sensibilidad al movimiento y al flujo.

confluencia de las venas pulmonares ${ }^{9}$.

En la tomografía con contraste se identificará una opacidad tubular, a veces toruosa, que representa la estructura venosa pulmonar dilatada. La resonancia magnética juega un papel emergente y puede diferenciar si la lesión es un aneurisma auricular o una variz venosa pulmonar, ya que una várice no estará cubierta de grasa pericárdica.

Actualmente la angiografía pulmonar con catéter selectivo es el estándar de oro para el diagnóstico de la variz pulmonar ${ }^{3}$, sin embargo, está reservada para estudios no concluyentes solo cuando una tomografía computarizada no puede proporcionar un diagnóstico claro. La ecocardiografía puede detectar várices cercanas al corazón, a la vez que pueden detectar la enfermedad de la válvula mitral que como se ha mencionado, tienen una asociación directa ${ }^{5}$.

En la mayoría de los casos, el tratamiento es innecesarios. Sin embargo, el monitoreo con estudios de imagen a diversos intervalos de tiempo es crucial. Para los pacientes con várices complicadas por aumento en su tamaño, hemoptisis, compresión esofágica o tromboembolismo, puede justificarse una intervención quirúrgica ${ }^{3}$.

Si una várice pulmonar se presenta como una masa central, redonda o lobulada en la radiografía de tórax, las consideraciones de diagnóstico diferencial pueden incluir cualquier causa de nódulo o masa pulmonar, linfadenopatías hiliares o mediastínicas ${ }^{10}$.

\section{Referencias}

1. Kumazoe H, Komori M, Ochiai R, Egashira R, Nakazono T, Kudo S. Pulmonary varix mimicking arteriovenous malformation. Clin Imaging. 2008; 32: 61-64.

2. Abujudeh $\mathrm{H}$. Pulmonary varix: Blood flow is essentials in the diagnosis. Pediatr Radiol. 2004; 34: 567-569.

3. James BG, Saketh PS, Felix H, Ramon V, Jinesh PM. Pulmonary Varix: An Uncommon Pulmonary Vascular Anomaly. Clin Pulm Med. 2017; 87-91.

4. Uyama T, Monden Y, Harada K, et al. Pulmonary varices: a case report and review of the literature. Jpn J Surg. 1998; 18: 359-362.

5. Nafees M, Abbas G, Amin MU. Pulmonary varicesrare cause of haemoptysis. J Coll Physicians Surg Pak. 2011; 21(7): 437-438.

6. Vanherreweghe E, Rigauts H, Bogaerts $Y$, Meeus L. Pulmonary vein varix: diagnosis with multislice helical CT. Eur Radiol. 2000; 10: 1315-1317.

7. Narula J, Talwar KK, Bharani A, et al. Pulmonary varix associated with mitral valve disease. Cathet Cardiovasc Diagn. 1987; 13: 411-413.

8. Dana Al, Reem Al, Usama Al, Claude PJ, Ebrahim $A E$. Pulmonary venous varix associated with mitral regurgitation mimicking a mediastinal mass: $A$ case 
report and review of the literature. Elsevier Radiology Case Reports. 2018; 404-407.

9. Berecova Z, Neuschi V, Boruta P, Masura J, Ghersin E. A pulmonary vein varix-diagnosis with ECG gated
MDCT, MRI and invasive pulmonary angiography. $J$ Radiol Case Rep. 2012; 6(12): 9-16.

10. Porres D, Morenza O, Pallisa E, et al. Learning from the pulmonary veins. RadioGraphics. 2013; 33: 999-1022.. 